\title{
Identificación de los pilares que direccionan a una institución universitaria hacia un smart-campus
}

\author{
Identification of the pillars that direct a university institution towards a smart- \\ campus
}

\author{
Claudia Jazmín Galeano-Barrera ${ }^{1}$ \\ Daniela Bellón-Monsalve² \\ Sergio Andrés Zabala-Vargas ${ }^{3}$ \\ Efrén Romero-Riaño ${ }^{4}$ \\ Viviana Duro-Novoa ${ }^{5}$ \\ Recibido: febrero 12 de 2018 \\ Aceptado: abril 23 de 2018
}

\begin{abstract}
Resumen
El presente trabajo expone los principales resultados de la primera fase de una investigación asociada a la determinación de un modelo general de un campus inteligente. Se realizó una revisión de la literatura a partir de una rigurosa selección de documentos, lo cual ha dadolugaral planteamiento de los pilares que dirigen la universidad a realizar prácticas inteligentes, integrando la gestión del conocimiento, la innovación y la sostenibilidad. El modelo flexibiliza la incorporación de elementos institucionales, respetando siempre la autonomía universitaria e inclusión social, con el fin de proponer un marco de trabajo que permita guiar a las Instituciones de Educación Superior hacia el desarrollo inteligente de forma integral que beneficie a toda la comunidad universitaria.
\end{abstract}

Palabras clave: universidades inteligentes, gestión del conocimiento, teoría de la innovación, sostenibilidad, prácticas inteligentes.

\begin{abstract}
This article makes public the main results of the first phase of a research associated with the determination of a general model of an intelligent campus. A review of the literature was made based on a rigorous selection of documents, which has led to the establishment of the pillars that direct the university to carry out smart practices, integrating knowledge management, innovation and sustainability. This model makes the incorporation of institutional elements more flexible, always respecting university autonomy and social inclusion, in order to propose a framework that allows higher education institutions to be guided towards an intelligent development in an integral way that benefits the whole university community.
\end{abstract}

Keywords: smart campus, knowledge management, innovation theory, sustainability, smart practices.

1 Ingeniera Industrial, Magíster en Desarrollo Sostenible y Medio Ambiente, Universidad de Santander, Bucaramanga, Colombia. E-mail: claudia.galeano@udes.edu.co

2 Ingeniera Industrial, Magíster en Sistemas Energéticos Avanzados, Universidad de Santander, Bucaramanga, Colombia. E-mail: dan. bellon@mail.udes.edu.co

3 Ingeniero Electrónico, Magíster en Administración de Proyectos, Universidad de Santander, Bucaramanga, Colombia. E-mail: Sergio. zabala@cvudes.edu.co

4 Ingeniero Industrial, Magíster en Ingeniería Industrial, Universidad Industrial de Santander, Bucaramanga, Colombia. E-mail: eromeror1483@gmail.com

5 Licenciada en Pedagogía, Máster en Gestión de la Información, Universidad Jesuita de Guadalajara, La Habana, Cuba. E-mail: viviana871007@gmail.com 


\section{Introducción}

En el mundo emergen continuamente prácticas que apuntan al mejoramiento de la calidad de vida de los ciudadanos, originadas a partir de la búsqueda de soluciones a grandes problemáticas tanto sociales, como económicas y ambientales; es por ello que la sostenibilidad, direcciona sus propósitos hacia la solución de necesidades que garanticen bienestar social sin comprometer las futuras generaciones. Dicho concepto, que se ha implementado a nivel empresarial e institucional, ha trascendido a nivel ciudad, donde se han incorporado otras variables que favorecen el desarrollo territorial, tal como lo son el uso de las Tecnologías de Información y Comunicación -TIC-, sistemas de movilidad y transporte, gobernanza, salud y demás que el contexto permite identificar. Es así que la Universidad de Santander -UDES-, reconociendo la importancia del desarrollo institucional y teniendo en cuenta su comunidad académica como eje principal para el desarrollo de sus labores, ha considerado determinar un modelo de Universidad Inteligente o Smart Campus. Dentro de las prácticas inteligentes que realiza una universidad, se encuentran la gestión y aseguramiento del conocimiento con la finalidad de crear, usar, validar, almacenar y transferir información y experiencias, desde que se originan hasta que se realiza su aplicación con el fin de generar valor. Dicha práctica, se encuentra en la cúspide de los pilares de un Smart Campus, puesto que es de gran importancia sumarle a la inteligencia empresarial, los datos que se encuentran en el contexto para la correcta toma de decisiones, logrando constituir una estructura empresarial innovadora y eficiente.

Como parte de estos Campus Inteligentes, es necesario hablar acerca del conocimiento, el cual corresponde a la acción y trae inmerso la aplicación personal, en valores y creencias. Es esto, lo que justamente lo diferencia de la información tomándolo como un objeto que existe independientemente de la persona, tal como lo menciona Liberona y Ruiz (2013), en su descripción de Gestión del conocimiento: los datos son el insumo para la estructuración de la información a la que se le adiciona sentido, significado, relevancia y propósito para transformarse en conocimiento, con el fin de lograr una aplicación personal e incorporar valores y creencias. El conocimiento, al igual que muchos otros elementos, existe al interior de las personas, por lo que forma parte de la complejidad e imprevisibilidad humana. Estas características son las que justifican que el conocimiento no sea un activo definible y tangible sino, por el contrario, intangible, difícil de capturar y gestionar (Martínez-Caro, 2009).

Una empresa puede conseguir unos resultados superiores basándose en su habilidad para generar nuevo conocimiento y utilizar la base existente de una forma más eficiente que sus competidores (Grant, 1996; Nonaka \& Takeuchi, 1995; Smith, Collins \& Clark, 2005). Para ello, las empresas crean las Estrategias para la Gestión del Conocimiento -EGC- tanto intrínseca como extrínsecamente, logrando incursionar de forma significativa en la competitividad empresarial por su generación de valor a través de las habilidades para la gestión del conocimiento. Es por eso que las EGC se consideran como un factor de creciente importancia para contribuir a que la empresa alcance ventajas competitivas a través de la innovación (Donate-Manzanares \& Guadamillas-Gómez, 2010).

Asimismo, las EGC son flexibles y adaptables al contexto empresarial donde, según la naturaleza de la organización, se plantean sus propias estrategias con objetivos e indicadores que, basados en autores como Gonzalez-Ariza, Castro y Roncallo (2004), deben contener seis aspectos o elementos dentro de su investigación, relacionada con la determinación del estado de la gestión del conocimiento en el contexto de cualquier organización: identificación del conocimiento, transmisión del conocimiento, medios y tecnología, toma de decisiones, cultura organizacional y competencia. Actualmente, la gestión del conocimiento se encuentra presente en diferentes sectores económicos, donde la academia es protagonista activa, incluso entes gubernamentales como el Ministerio 
de Educación Nacional han generado un desafío alrededor del tema (Ministerio de Educación Nacional, 2017).

Teniendo en cuenta lo anterior, el estudio de las relaciones entre la innovación tecnológica y la Gestión del Conocimiento Organizativo -GCO- se considera un campo de investigación novedoso en el ámbito de la Dirección de Empresas (Nieto-Antolín \& Navas-López, 2003). Así, la teoría de la innovación juega un papel fundamental en la generación de conocimiento, debido a la obtención de datos y experiencia, los cuales posteriormente se operativizan, con el fin de realizar una implementación social de los mismos. Asimismo, cuando se habla de innovación implica necesariamente hablar de un cambio y una evolución, tal y como se observa en la transición acontecida entre los términos de innovación cerrada y la innovación abierta mencionadas por Shumpeter, mientras que Chesbrough afirma que en la actualidad se debe ir mucho más allá de los límites internos de una organización y la intervención de entes externos tienen un papel relevante para su desarrollo (Jazmín, Barrera, Romero \& Becerra Ardila, 2015). En este sentido, la innovación se constituye como eje fundamental para el crecimiento empresarial, buscando ventajas competitivas y el fortalecimiento de sus capacidades para la mejora continua.

Por otra parte, al hablar de innovación tecnológica y cooperación entre agentes económicos y sociales como motores de cambio, se realiza un acercamiento a marcos de trabajo internacionales, como los principios de desarrollo sostenible impulsados por la ONU en el programa 21, que se encaminan hacia aspectos como:

- La infraestructura tecnológica: promoción de las TI, redes de información como mecanismo de comunicación, plataformas inteligentes, infraestructuras e coeficientes.

- La gestión energética: uso eficiente de energías renovables, sistemas de almacenamiento y aprovechamiento de energía.
- La gestión y protección de los recursos: planificación del territorio y de los recursos basada en criterios de sostenibilidad y cooperación entre administraciones.

- La provisión de servicios: desarrollo de nuevos modelos colaborativos que permitan integrar lo público y lo privado, interoperabilidad de procesos y servicios.

- El gobierno: accesibilidad e interoperabilidad de los datos, transparencia en la gestión, aplicación de políticas sostenibles.

Lo anterior indica que la temática de investigación se relaciona con áreas determinadas como estratégicas para el desarrollo internacional, con el fin de impulsar el cambio que genere escenarios más favorables para las generaciones futuras. Alineado con los anteriores principios, existe otro factor fundamental para la generación y conservación de la innovación en las organizaciones, correspondiente al recurso humano, puesto que al establecer una cultura en los colaboradores, se fomenta la aceptación de la innovación como valor fundamental en la organización, comprometiéndose esta misma (Hartmann, 2006; Naranjo-Valencia, Jiménez-Jiménez \& Sanz-Valle, 2012). De lo anterior se identifica la importancia y la necesidad de determinar los pilares que soportan un Campus Universitario con el fin de reconocerlo por sus prácticas inteligentes, de tal manera que pueda obtener una ventaja competitiva frente a otras Instituciones del Sector y garantice la sostenibilidad en un ámbito social, ambiental y económico.

\section{Materiales y métodos}

Para el desarrollo de la presente investigación se definieron tres etapas fundamentales, las cuales se relacionan en la Figura 1. 


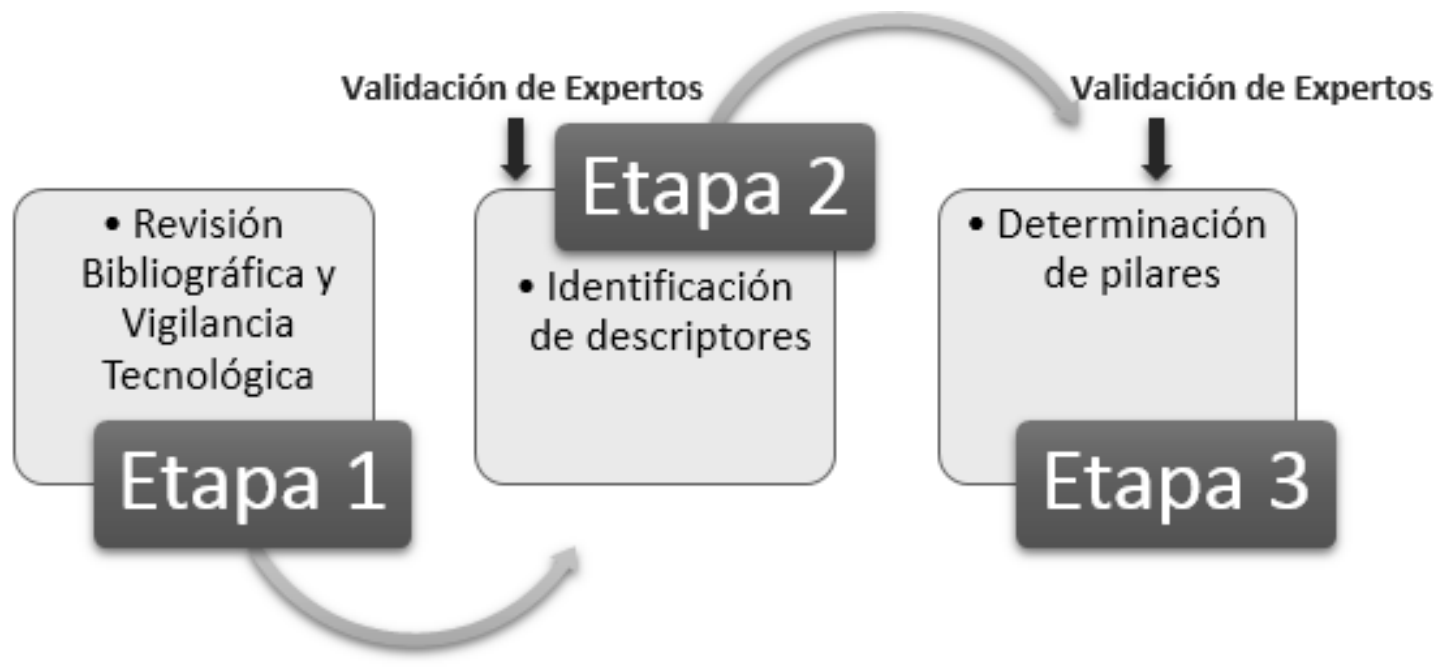

Figura 1. Etapas metodológicas de la investigación.

El estudio inicia con la etapa 1, para la cual se tomó como base un ejercicio de revisión bibliográfica y de vigilancia tecnológica, reconociendo ésta última como una función fundamental para la planificación, gestión y desarrollo de proyectos (Muñoz-Durán, Marín-Martínez \& Vallejo-Triano, 2006). Dado que existen diversas metodologías para el ejercicio de vigilancia tecnológica, se adoptó el protocolo establecido por Vargas y Castellanos (2005), en el cual se plantea un proceso ordenado de pasos que se ajusta al objetivo de la investigación, correspondientes a planeación, preparación para la búsqueda, ejecución de la búsqueda en la base de datos, depuración y convalidación de registros. En ésta etapa se identificaron artículos, patentes y casos de estudio a nivel internacional, los cuales sirvieron como apoyo a la determinación de los pilares fundamentales, que influyen en el direccionamiento de una Institución Universitaria hacia un Campus Inteligente o Smart Campus. A continuación, se describe el protocolo desarrollado:

Planeación: Este proceso inicia con el desarrollo de un conversatorio entre un grupo interdisciplinario, conformado por profesionales en áreas de la ingeniería, informática, diseño gráfico y arquitectura. Como resultado, se obtuvo el punto de vista desde las diversas áreas sobre prácticas Inteligentes al interior de las Universidades, que al momento se llevan a cabo de forma desarticulada y no sistematizada, emergiendo una variedad de temáticas. En éste sentido, se define la realización de una revisión exploratoria.

Preparación para la búsqueda: se usan fuentes secundarias de información y refinación de artículos científicos de bases de datos estructuradas. Al iniciar la fase exploratoria, se usó como patrón de búsqueda "Universidades Inteligentes" pero no se obtuvo resultado concluyente, dado que no existían publicaciones en español, pero sí en inglés, lo que conllevó a descubrir qué subtemas se derivan de éste tipo de Instituciones. Para ésta fase se trabajó con las bases de datos de Scopus y la ISI Web of Science.

En la segunda fase de la figura 1, se usaron las siguientes ecuaciones de búsqueda: $\mathrm{i})$ (TS=((smar*universit*model)OR(smar* camp*)OR(ambien*intelligenc*)AND(enviroment*)(economic*)(social*) OR(Sustainabl*)AND(internetofthings)OR(ICT)AN$D$ (citizen culture) AND (critical sucess factors) OR (key factors* $)))$. li) $\left(\left(\mathrm{TS}=\left(\left(\left(\right.\right.\right.\right.\right.$ smar* $^{*}$ universit*model) OR(smar*camp*))OR((ambien*intelligenc $\left.{ }^{*}\right)$ OR(smartgrids)OR(sustainable))OR((internet of things) OR(web of things)OR(technology))OR ((communit*)OR (societ*))))). lii) TS= ("smart university" OR 
"intelligent university" OR "smart campus" OR "intelligent campus").

Búsqueda en la base de datos: usando las ecuaciones de búsqueda seleccionadas, se identificó que la primera y segunda ecuación conducían a una búsqueda desviada de la temática principal de investigación, por tanto, se usó la tercera ecuación como elemento definitivo, tomando como ventana de tiempo del año 2006 al 2016, la cual arrojó los resultados conforme a la temática de interés. Posteriormente, se refinaron los artículos encontrados seleccionando 12 , de los cuales 2 pertenecen al año 2008, 3 al año 2015 y 7 al año 2016.

Depuración y convalidación de registros: el proceso de revisión de artículos permitió determinar los descriptores comunes, usados en la selección de variables de mayor influencia en el proceso de orientación de una Institución Universitaria hacia un Smart Campus. Dichos descriptores constituyen un elemento clave dentro de la determinación de los pilares planteados en el presente trabajo.

En la segunda etapa se identificaron los descriptores, reconociendo la importancia de los mismos dentro del proceso de determinación de los pilares objeto del presente trabajo. El cumplimiento de la fase se dio a partir del análisis de los resultados obtenidos en la etapa 1. La tercera etapa permitió determinar los pilares fundamentales en el direccionamiento de una Institución Universitaria hacia un Smart Campus. Dicho proceso fue producto del análisis de los resultados de las etapas 1 y 2; así como de la validación de los resultados previos por un grupo de expertos en la temática y realizando una integración con búsqueda de patentes usando frecuencia de palabras, así como los principios de la gestión del conocimiento y casos de estudio.

\section{Resultados y discusión}

Inicialmente es necesario revisar el término Making Smart o hacer inteligente, el cual se centra en dos aspectos: i) el conocimiento profundo de un área y ii) la elección de acciones y estrategias evaluadas con base a ese conocimiento (Mattoni et al., 2016). Este concepto supone la aparición de un método inteligente que incluye características en detalle, y una percepción e inteligencia profunda (Tan, 2006). Con base en el anterior concepto y el método inteligente, se construye el modelo de sistema inteligente, como un conjunto integrado que incorpora sistemas de automatización, para proporcionar un sofisticado monitoreo y control sobre las funciones de los componentes por medio de dispositivos electrónicos. Por ejemplo, un sistema doméstico inteligente puede controlar la temperatura, la iluminación, los medios múltiples, la seguridad, entre otras funciones (Khabou, Rodriguez, Gharbi \& Jmaiel, 2014). Dentro de este paradigma, emerge el concepto de "Smart Systems", compuesto por materiales y tecnologías integrados para superar los requisitos de desempeño tradicionales (Hipwell, 2014). En paralelo con los "Smart Systems", los "Intelligent Systems" adicionalmente pueden razonar, planificar y aprender. Los sistemas inteligentes no sólo poseen la capacidad de hacer ajustes, sino también pueden aprender sobre los usuarios y su entorno, así como adaptarse a los cambios.

En ese sentido, el libro blanco de "Intelligent Campus" iCampus, define un grupo de indicadores clave de desempeño basados en los pilares de iCampus. Estos indicadores clave de rendimiento son: (a) la reputación de la institución; (b) la calidad del aprendizaje y la enseñanza; (c) el impacto y desempeño de la investigación; (d) el ambiente educativo y el compromiso. De esta forma, se mide la significancia del avance del modelo de iCampus en las diferentes instituciones (Aion, Helmandollar, Wang \& Ng, 2012).

Los campus universitarios pueden considerarse complejos sistemas compuestos por varios elementos, como: usuarios, edificios e infraestructuras, entre otros, constantemente influenciados entre sí (Mattoni et al., 2016). Los sistemas Smart Campus, proporcionan diferentes servicios a sus grupos de interés, para mantener la cooperación 
entre alumnos y profesores a través de dispositivos conscientes de su contexto ambiental. De esta forma, los Campus Inteligentes habilitan una infraestructura física y tecnológica que permite que los dispositivos y sistemas sean controlados y adaptados de forma automática según el contexto (Makdissi, Boutaud \& Dalmolin, 2016). Durante los años 50 , se establecieron en Latinoamérica modelos de infraestructura física de las universidades basados en conceptos de urbanismo y de ciudades inteligentes, por lo cual, es común la denominación de los campus como ciudad universitaria. Igualmente, el modelo de los campus inteligentes ha sido influenciado por las funcionalidades y los conceptos de ciudades inteligentes.

Conceptos como la autonomía para los consumos eléctricos y térmicos, mediante el uso combinado de centrales térmicas y de energías renovables enviadas a través de un control de software central, puede convertirse en un ejemplo de estrategia de ciudad, viable para campus universitarios (Lazaroiu, Dumbrava, Costoiu, Teliceanu \& Roscia, 2015) y aplicable a nivel mundial. De acuerdo con (Mattoni et al., 2016), las principales dimensiones de un Smart Campus son: educación y conocimiento, comunicación, transporte y gobernanza. Con base en estos antecedentes conceptuales, a continuación se abordan modelos y marcos de trabajo de Smart Campus.

\subsection{Modelos y marcos de trabajo}

La similitud entre los campus universitarios y las ciudades, ha sido la fuente de extensión e intercambio de conceptos entre estos dos espacios territoriales tan diversos. El modelo urbano de Smart City es uno de los más difundidos y de amplia aceptación, en parte por su naturaleza sistémica y multidimensional, construida con base en la participación de los ciudadanos. El modelo de Smart City propuesto por Mattoni et al. (2016), se compone de seis aspectos urbanos principales: Gobernanza, Gente, Economía, Medio Ambiente, Vivienda y Movilidad. Estas ciudades inteligentes se diferencian por ofrecer servicios a sus habitan- tes con el fin de facilitar la vida cotidiana (Szabo et al., 2013). Desde la perspectiva del conocimiento y la información, Gartner definió las ciudades inteligentes como "múltiples sectores que cooperan para lograr resultados sostenibles a través del análisis de la información contextual en tiempo real, compartida entre la información sectorial específica y los sistemas de tecnología operacional". Estos enfoques se complementan para enriquecer y generar los modelos de Smart Campus o Campus Inteligente.

De acuerdo con Atif y Mathew (2013), un campus inteligente proporciona conectividad entre los estudiantes y sus entornos circundantes, integrando la gente con los recursos físicos. El éxito de un Campus Inteligente radica en su capacidad para conectar comunidades, basado en vínculos sociales que existen entre sus miembros. En ese sentido, derivado de las dimensiones de las ciudades inteligentes, se formula un modelo de Smart Campus como un conjunto multidimensional compuesto por: i) personas y hábitat, ii) economía, iii) energía, iv) medio ambiente, iv) y movilidad. A continuación, se define cada uno de los campos de acción de Smart City orientado hacia Smart Campus:

La dimensión de personas y hábitat se orienta hacia la actuación del campus desde puntos de vista académico, de investigación y de gestión administrativa, la oferta y calidad de los servicios primarios y secundarios, así como la asequibilidad económica.

La dimensión de economía comprende la evaluación de los planes económicos, la calidad y los objetivos de las inversiones, la asignación equilibrada de la financiación pública, apoyo a la innovación tecnológica y creación de spin-off y colaboraciones interuniversitarias, entre otras.

La dimensión de energía se enfoca en la producción, distribución y consumo de energía. La evaluación del uso de fuentes renovables considera la identificación y el uso de energía; la producción; las redes de distribución, fugas de edificios y espa- 
cios al aire libre, arquitecturas bioclimáticas y medidas de consumo de energía y monitoreo.

La dimensión de medio ambiente comprende el impacto en términos de contaminación, promoción y protección de zonas verdes, gestión de aguas residuales, uso de recursos y microclima de interiores y exteriores, y calidad del aire.

El componente de movilidad tiene como objetivo evaluar los sistemas de transporte y logística hacia, desde y dentro del campus. El campo de la movilidad en los Smart Campus se analiza desde distintos puntos de vista: infraestructural, redes (parqueaderos, centros de conmutación, acceso y accesibilidad, zonas peatonales, etc.), redes de vehículos y ciclistas (transporte público, compartir bicicleta).

Los campus universitarios representan un ejemplo perfecto de las células urbanas y son comparables a las ciudades, a pesar de las diferencias en la dimensión y tipo de estructuras. Una de las razones por las cuales los marcos de trabajo de ciudad inteligente son extendibles al estudio de campus inteligente, es por que comparten desafíos tales como los costos de energía, la aglomeración, las conexiones y accesos, movilidad y estacionamiento, y una oferta insuficiente de servicios de valor para los usuarios o habitantes (Pagliaro et al., 2016).

En ese orden de ideas se presenta a continuación, hallazgos derivados de un análisis comparativo entre modelos de ciudad y campus inteligente. Para este análisis se toma como referencia los seis ejes de desarrollo de una universidad inteligente que postula: Gobernanza, Gente, Economía, Ambiente, Habitabilidad y Movilidad, socializado en Centre of Regional Science (2007).

En síntesis: i-) Gente y habitabilidad: incluye acciones relacionadas con el concepto más amplio de Smart Community. Las intervenciones pretenden reorganizar los servicios existentes y suministrar nuevos servicios con el fin de mejorar la calidad de vida de los usuarios, ii-) Economía y Gobernanza: se sugieren acciones para apoyar el desarrollo de planes de economía inteligente en este campo. De esta manera, la gobernanza universitaria puede mejorar sus dotaciones y apoyar la innovación en el desarrollo de productos y servicios, iii-) Energía: estas acciones están dirigidas a mejorar la producción, distribución y consumo de energía dentro del campus, con el fin de aumentar el rendimiento energético de los edificios, entre otros, iv-) Medio ambiente: acciones dirigidas a mejorar la calidad del aire interior y exterior, optimizando el consumo de elementos naturales y v-) Movilidad: se trata de la optimización de la movilidad pública y privada hacia, desde y dentro del campus. Se orienta a disminuir el uso de vehículos privados y la implementación de soluciones sostenibles.

\section{Aproximación al concepto de Smart-campus desde un ejercicio de vigilancia tecnológica}

El proceso de revisión bibliográfica y vigilancia tecnológica, constituye el elemento clave del trabajo llevado a cabo, ya que como afirman Vargas y Castellanos (2005), su objetivo es la recopilación de datos, los cuales posteriormente son transformados mediante un proceso de clasificación, análisis y relación con el entorno, que logran generar un sistema de inteligencia. En éste sentido, se evaluó en primera instancia las publicaciones realizadas en la materia de investigación; permitiendo identificar que la temática ha evolucionado lentamente a través del tiempo desde el año 2008 donde sólo se generaron dos publicaciones, hasta el año 2016 donde se publicaron 7 artículos; asimismo, fue posible identificar que los países pioneros en la investigación en ésta línea han sido Italia, Lituania, China, España y Arabia, con tasas iguales de participación. Por su parte, los autores de los artículos revisados, integran como elementos fundamentales de las Universidades Inteligentes las plataformas móviles, los ambientes inteligentes, la gestión ambiental y la gestión universitaria con prácticas inteligentes (Bures, Tucnik, Mikulecky, Mls \& Blecha, 2016; Tan \& Wang, 2010). 
En segunda instancia, se evaluaron las patentes generadas en torno a las universidades inteligentes mediante el software libre PATENTSCOPE, el cual proporciona acceso a las solicitudes internacionales del tratado de cooperación en materia de patentes en formato de texto completo el día de la publicación, y a los documentos de patentes de las oficinas nacionales y regionales de patentes participantes. La información puede buscarse introduciendo palabras clave, los nombres de los solicitantes, los datos de la Clasificación Internacional de Patentes y muchos otros criterios de búsqueda en varios idiomas.

Para iniciar la búsqueda se han usado las palabras que han generado mayor impacto y resultados directo con búsquedas de artículos las cuales son "Smart Campus", donde se ha obtenido un resultado de 6 patentes, generadas entre los años 2013 (1 patente) y 2014 (5 patentes); asimismo, se identi- ficó que la empresa Samsung ha sido la compañía líder en el apoyo de la generación de patentes, las cuales han tenido como factor común la aplicación tecnológica en el uso de tarjetas y red para control y disposición de datos (Información).

Como tercer elemento, se analizaron los casos de estudio en diferentes instituciones educativas a nivel internacional, a partir de experiencias y proyectos asociados al concepto de Universidades Inteligentes. Como resultado de éste ejercicio, se encontró que las propuestas cada vez recopilan más datos de los usuarios, evidenciando la importancia de contar con soluciones para el procesamiento de las mismas a través de herramientas que permitan adecuar la gestión de las universidades en su totalidad, conforme a los requerimientos reales de los usuarios. La Tabla 1 relaciona los casos de estudio identificados.

\section{Caso de estudio}

Proyecto/comunidad

Hydra (Hydra, 2017)

Proyecto (Stavropoulos, Koutitas, Vrakas, Kontopoulos Vlahavas, 2016)

Proyecto de universidad/ ciudad inteligente en Hungría. (R. Szab et al., 2013)

Propuesta desarrollada en la República Checa. (Vladimír Bureš, Petr Tučník, Peter Mikulecký, Mls \& Blecha, 2016)

\section{Descripción}

Proyecto implementado desde 2008 por las Universidades de Stanford, Virginia y Hull. Este fue creado para ser un repositorio multipropósito de aplicaciones, marcos de referencia, herramientas de administración, entre otros; orientado a las necesidades universitarias. Actualmente cuenta con más de 30 instituciones a nivel internacional que contribuyen con aplicaciones, software, diseños, metadatos, herramientas tecnológicas, entre otros, para uso en comunidad.

Desarrollado en la IHU, International Hellenic University, Grecia, presenta la base tecnológica para la integración de los elementos conceptuales de las universidades \& inteligentes con las herramientas tecnológicas requeridas. Técnicamente, se diseñó una herramienta Middleware (interfaz entre hardware y software) basada en servicios Web y aplicativos como Idealims y PlugDroid, ambos diseñados para el monitoreo y ahorro de energía en los recintos universitarios (Stavropoulos, Gottis, Vrakas \& Vlahavas, 2013). El aplicativo se continúa desarrollando con el estándar OpenADR, logrando reducciones mayores al 16\% del consumo energético de la institución.

Integra el concepto de participatory sensing. Se propone un marco de trabajo que se fundamenta en el análisis en tiempo real de la información proveniente de mediciones distribuidas. Particularmente, en el caso del i-campus se cuenta con un servicio web que permite, además del monitoreo de información de la comunidad, la difusión de eventos, el desarrollo de actividades académicas, la ubicación de los miembros según ventanas de tiempo, entre otros.

Permite observar cómo, a través del concepto de la inteligencia ambiental, es posible realizar asistencias inteligentes para la preparación de trabajos de grado de estudiantes, la gestión de videoconferencias y la administración de campus universitarios. Particularmente se presentan las arquitecturas tecnológicas requeridas para cumplir dichos propósitos. 


\begin{tabular}{|c|c|}
\hline Caso de estudio & Descripción \\
\hline $\begin{array}{l}\text { Proyecto de Smart Campus } \\
\text { en la University of Science } \\
\text { and Technology of China. } \\
\text { (G. Sun, Y. Zhou \& J. Li, } \\
\text { 2016) }\end{array}$ & $\begin{array}{l}\text { Se ha venido desarrollando desde } 2012 \text {, iniciando con control de acceso a estudiantes, } \\
\text { administración de información y monitoreo/vigilancia con cámaras y sensores. } \\
\text { Actualmente, se enfrentan al monitoreo de comportamiento de la comunidad y sus } \\
\text { espacios de interacción. Se resalta el reto del manejo de la información y el cuidado de } \\
\text { los datos personales. }\end{array}$ \\
\hline $\begin{array}{l}\text { Proyecto en Institución } \\
\text { de Educación Superior } \\
\text { en China (Yunchuan et al, } \\
\text { 2014) }\end{array}$ & $\begin{array}{l}\text { Los autores establecen un marco de trabajo que facilita la dinámica de relacionar } \\
\text { eventosysus conexiones (asociadoa usuarios) en una institución de educación superior } \\
\text { en China. Con esta herramienta se puede estimar tendencias de la comunidad, sus } \\
\text { principales desplazamientos, relaciones causa-efecto, actividades secuenciales más } \\
\text { comunes, entre otros. Esto permite adecuar la administración y gestión del campus } \\
\text { según sus requerimientos e intereses. }\end{array}$ \\
\hline
\end{tabular}

Tabla 1. Casos de estudio asociados a Universidades Inteligentes.

Finalmente, vale la pena indicar que el concepto de Smart es relativamente nuevo y ha sido interpretado de muchas maneras diferentes durante su evolución. Dicho término incluye metodologías de planificación para mejorar los procesos urbanos, desarrollar estrategias de planificación yaplicar nuevas soluciones de manera coordinada y holística. Esta planificación integrada es el núcleo del concepto Smart, el cual puede aplicarse a diferentes escalas territoriales como las áreas regionales y las zonas urbanas (Pagliaro et al., 2016). Los resultados de planificación de ciudades o regiones, pueden ser evidenciados en ciudades emblemáticas como Bilbao, donde se implementó el Urban Project Bilbao, Ria Bilbao 2020, entre otros. En este sentido, se incluye el componente de planeación inteligente y participativa como pilar del marco de trabajo de esta investigación. En efecto, un proyecto inteligente debería tener en cuenta no sólo el desarrollo de las tecnologías de la información y la comunicación (TIC), sino también centrarse en los aspectos infraestructurales, culturales y funcionales, así como las necesidades de los usuarios finales. Además, un proyecto inteligente o una acción inteligente debe ser replicable y adaptable a otro contexto para ser realmente eficaz. Para alcanzar este objetivo, debe realizarse un análisis preliminar de las características y necesidades sociales y estructurales (Pagliaro et al., 2016).

\subsection{Identificación de descriptores}

\section{Análisis en software Nvivo}

Para la identificación de descriptores se usó el software NVivo, especializado para investigaciones cualitativas y mixtas, mediante el cual se realizó un análisis de datos a partir de la frecuencia de palabras de los artículos hallados en la búsqueda inicial, el cual permitió analizar resultados, redes y aglomerados. A partir del software se revisó la frecuencia de palabras para obtener la marca de nube y el mapa ramificado, de donde se observa que la Universidad, la inteligencia y la proyección son el punto de partida de un grupo de palabras claves que se encuentran inmersas en los objetivos propuestos en la presente investigación y lo cual es un buen indicador. Palabras como aplicaciones, ambiente, conocimiento, agentes, administración, partes interesadas, cambio, lugares, resultados y otras integran el concepto de Smart Campus o Smart University.

Con la información obtenida acerca de las patentes, se realiza el mismo ejercicio para la frecuencia de palabras y mapa ramificado, encontrando que dentro de las palabras más usadas y comunes entre la descripción de las patentes se tiene a la Información como factor clave que genera dispositivos, métodos, aparatos, paquetes de datos, trabajo en red, así mismo el 
uso de plataformas, sistemas y contenidos que permiten un mejor flujo de información y por ende de comunicación. Por último, se realiza el análisis mediante el cruce de frecuencia de palabras entre los artículos con las patentes encontradas, con el fin de obtener semejanzas y un análisis de aglomerados que se evidencian en la Figura 2. Al analizar los resultados, tanto de revisión de literatura como de patentes sobre el mismo tema: "Smart Campus", se puede observar que los autores determinan en común 4 elementos fundamentales, correspondientes a la Inteligencia (Smart), la información, la Universidad, y el conjunto o paquetes que contemplan tecnología, ambiente, equipos, lugares y personas.

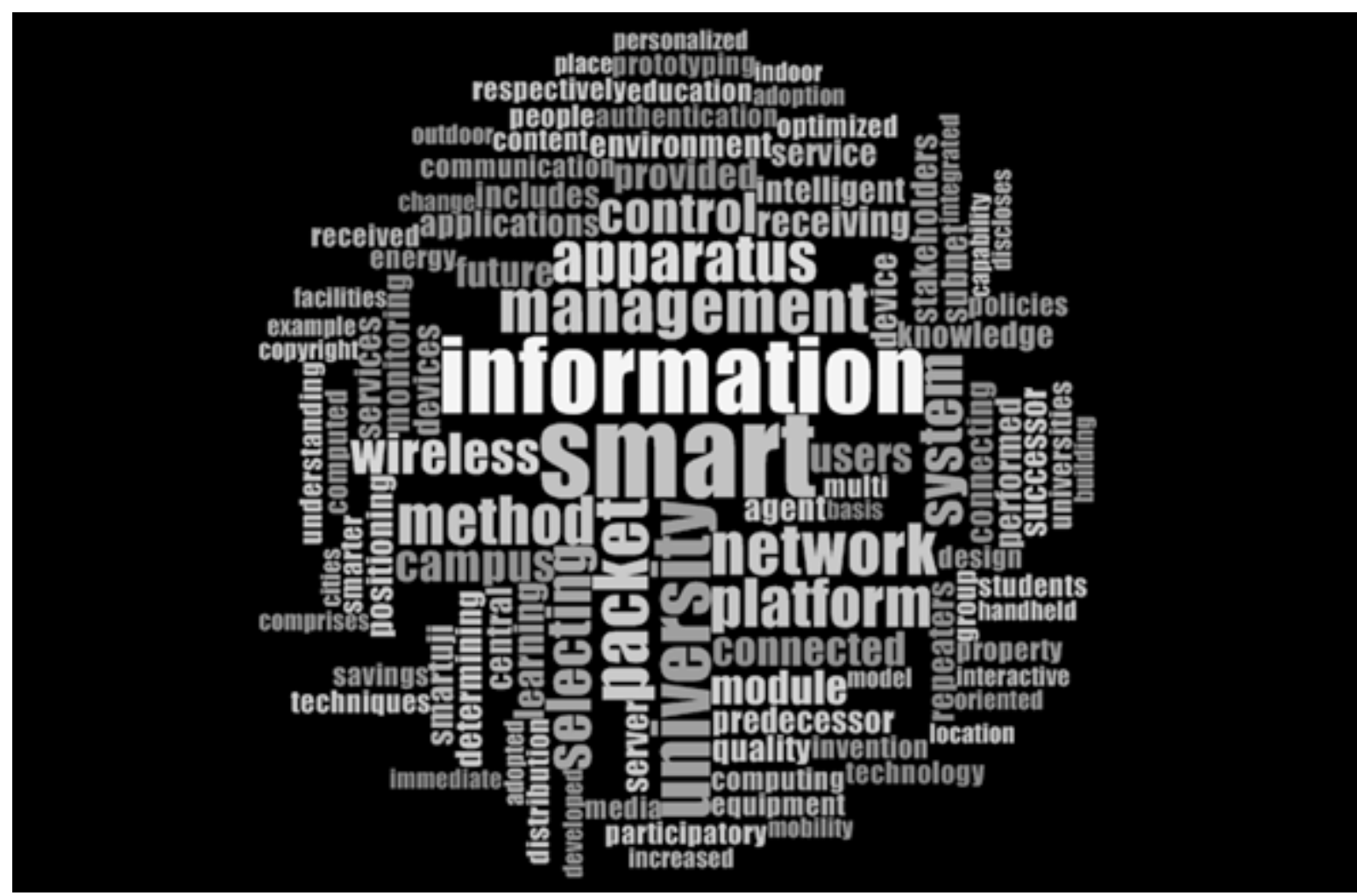

Figura 2. Palabras clave de artículos vs Patentes.

Respecto a Smart, ésta se concibe como un elemento que se relaciona principalmente con la administración, conectividad, sistemas y usuarios; la información principalmente se enlaza a la red o conectividad, aparatos y dispositivos, sistemas, control y cambio tanto interno como externo a la organización; el pilar de Universidad se enfoca a los métodos aplicados en el campus, red inalámbrica, dispositivos, gestión ambiental, energía, servicios, derechos de autor y cambio; y por último, en cuanto al factor "packet", éste se conecta con uso de plataformas, interacción con el campus, conectividad, desarrollo sostenible, partes interesadas, lugares y facilitadores.

\section{Determinación de descriptores}

El concepto de Universidad Inteligente, Smart Campus por sus siglas en inglés, cuenta con diferentes definiciones en la literatura. Por ejemplo, Berghofer (2013), indica que: "La Universidad Inteligente es la visión de la universidad como una plataforma que adquiere y entrega datos fundamentales para impulsar el análisis y la 
mejora del entorno de enseñanza y aprendizaje" (p.2). El proyecto Catapult-Future Cities, soportado por la Universidad de Glasgow, define el concepto como aquel que aprende activamente y se adapta a las necesidades de su gente y lugar, desvelando el potencial de la tecnología y permitiendo un aprendizaje y una investigación que cambian el mundo (Catapult-Future Cities, 2016). Otros aspectos que deben ser tenidos en cuenta al definir Campus Inteligentes se asocian a leyes, políticas, aspectos económicos, análisis de mercados, relaciones entre actores, y muchos otros procesos de una organización (Coccoli, Guercio, Maresca \& Stanganelli, 2014)

Ahora bien, sin duda las herramientas tecnológicas contribuyen a que los elementos generales de la educación en un ambiente inteligente, prosperen. Es en este marco tecnológico, donde se reconoce a las redes de próxima generación (NGN) se encuentra no solo aspectos educativos sino también de comunicaciones, interacción social, transporte, administración, seguridad, salud, gobernanza, infraestructura tecnológica, manejo energético, gestión de información, entre otros. Cada uno de estos elementos se consolida en diferentes marcos de trabajo propuestos en la literatura, como el expuesto por Hirsch y $\mathrm{Ng}$, 2011). De acuerdo con lo anterior, así como con los cuatro elementos fundamentales establecidos en el análisis de Nvivo, se definen los descriptores de la Figura 3, los cuales representan la base de determinación de los pilares fundamentales que direccionan una Institución Universitaria hacia un Smart Campus, objeto de la presente investigación.

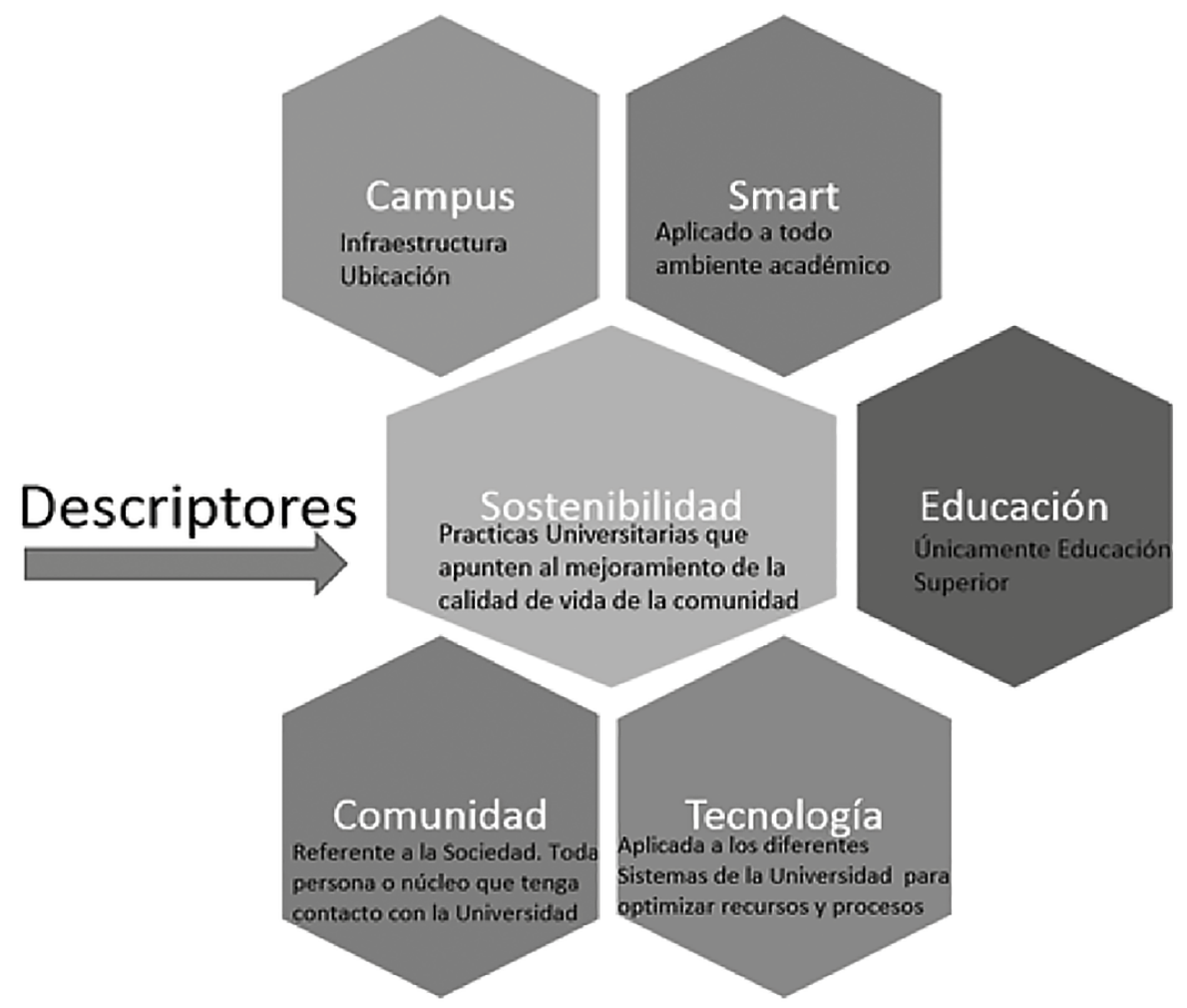

Figura 3. Descriptores relacionados con Smart Campus. 


\subsection{Determinación de pilares}

Después de realizar el ejercicio de selección de artículos y búsqueda de patentes, se procede a revisar la frecuencia de palabras y jerarquía, de tal manera que se obtiene una nube de palabras, las cuales fueron clasificadas por grupos, dando lugar a la identificación de los descriptores que componen un Smart Campus. Lo anterior, permite determinar los pilares que orientan las Instituciones Universitarias hacia un Smart Campus, delimitando las variables asociadas a cada pilar. Asimismo, dichas variables se soportan en la seguridad e incorporación de tecnologías; reconociendo que toda acción debe tener el sello de la innovación, el cual finalmente generará valor agregado para robustecer la Gestión del Conocimiento de la Universidad.

La determinación de los pilares se sustenta en el hecho de que las Universidades Inteligentes integran actores, estrategias, procesos y resultados, buscando una proyección hacia el mejoramiento continuo en la línea de tiempo, de tal manera que la inteligencia se encuentre inmersa en las acciones realizadas a través de diferentes dispositivos, prototipos, tecnología, modelos y prácticas. Es así que, se permite la articulación de las partes interesadas en búsqueda de fortalecer el conocimiento, procediendo sobre la sostenibilidad aplicada al campus, tal como se hace en las ciudades Inteligentes. Por último, es importante tener en cuenta la infraestructura, accesibilidad, agentes ambientales, lugares propicios para el bienestar de la comunidad, el compromiso y gestión de la administración como factor de éxito en los modelos de Universidades Inteligentes, dado que son escenarios propicios de participación activa de los agentes que integran la Universidad, de tal manera que como sistema se retroalimente y mejore continuamente. En éste sentido, los pilares determinados conforme el ejercicio expuesto en el presente trabajo, se encuentran centrados en:
- Educación: La cual tiene en cuenta el aprendizaje, la información y los datos.

- Ambiente: Involucra el uso eficiente de los Recursos Naturales, los sistemas de monitoreo ambiental, el manejo y disposición de los desechos finales y el Plan de protección de los recursos naturales.

- Sociedad: Actor fundamental para la operacionalización de las prácticas inteligentes en el cual se incluyen las redes sociales, la participación ciudadana, los patrones de conducta y el trabajo colaborativo.

- Gobernanza: Identificado como factor clave para el cumplimiento de un plan referente a Smart campus, incluye Políticas Internas, definición y estandarización de Procesos, certificaciones de calidad, acreditación, administración horizontal, liderazgo, motivación organizacional y Responsabilidad Social Universitaria.

- Infraestructura y Movilidad: Involucra vías de acceso al campus, fachadas inteligentes, construcciones inteligentes, movilidad dentro del campus.

- Salud: Tiene en cuenta principalmente la salud preventiva, programas de cuidado personal, APP para asesorías médicas, índice de seguimiento a pacientes y articulación con I+D+i para ejecución de investigaciones médicas.

- Investigación, Desarrollo e Innovación (I+D+i): Direccionado principalmente al Diseño, desarrollo y prototipado.

La Figura 4 muestra el esquema de los pilares, los cuales se soportan en tres elementos: la tecnología, la seguridad y la inteligencia, enmarcados dentro de la gestión de la innovación, lo cual repercute al final sobre la gestión total del conocimiento al interior de las instituciones universitarias. 


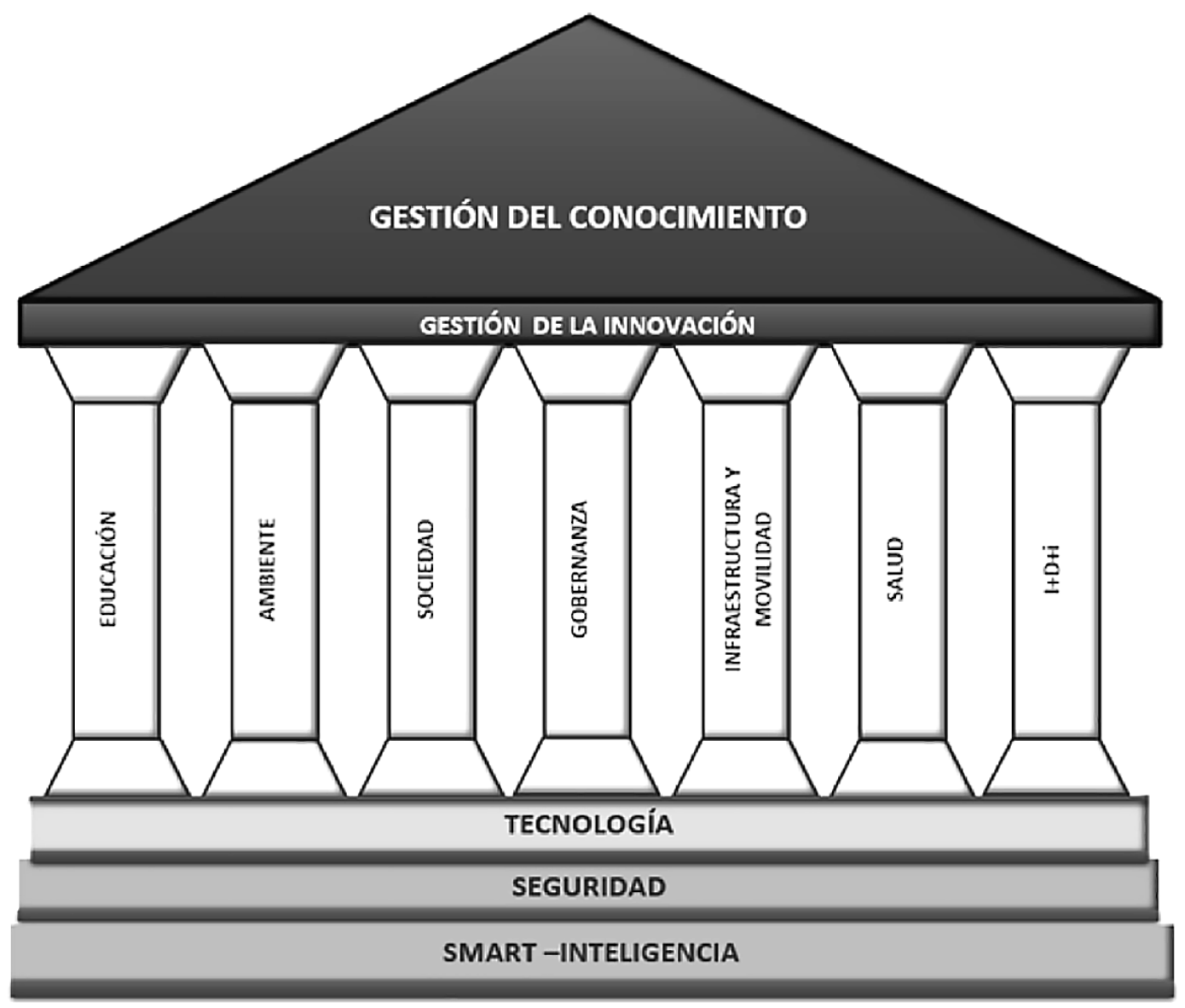

Figura 4. Pilares de un Smart Campus enmarcados en la Gestión del Conocimiento.

Con la determinación de los pilares enmarcados en la Gestión del conocimiento, la innovación, el factor tecnológico y la seguridad, se garantiza obtener las habilidades para generar soluciones inteligentes del campus universitario, por medio de prácticas diferenciadoras. Es por esto que lo anterior se soporta en la Inteligencia, la cual consiste en recopilar datos para ser clasificados y analizados, a fin de alcanzar una transformación que los articule a un entorno; es así como la inteligencia resulta ser una habilidad que integra la experiencia, logrando obtener conclusiones más fiables y tomar decisiones acertadas. Por otra parte, la Tecnología como base de los pilares de un campus inteligente juega un papel fundamental para el empoderamiento de la comunidad universitaria, ya que se busca que esta sea compatible con la sociedad, partiendo desde los espacios académicos (Pitre, Gómez \& Pertuz, 2017) que permitan la participación de los diferentes actores del entorno en la construcción de innovación educativa (Prettel, Cantillo \& Ávila, 2014) desde la gestión estratégica tanto de áreas administrativas de la organización a partir de variables endógenas y exógenas en busca de un aumento de la competitividad y la efectividad como dinamizadores del cambio cultural (Bustamante-Zapata., Porto-Pérez \& HernándezTaboada, 2013; Pertuz-Peralta \& Perez-Orozco, 2015), como del componente académico de las instituciones compuesto principalmente por docentes y estudiantes, donde cada uno de ellos acompañe su interacción con el uso adecuado de la tecnología para la generación de capacidades y 
se genere una formación integral e interdisciplinar (Valdelamar-Zapata, Ramírez-Cruz, RodríguezRivera \& Morales-Rubiano, 2015).

Asimismo, partiendo de la relación entre los componentes base (Tecnología, Seguridad e Inteligencia), se requiere de la flexibilidad y la adaptación al cambio organizacional (GarcíaRubiano \& Forero-Aponte, 2015) debido a la enorme cantidad de información que diariamente se genera, la cual repercute en variaciones a nivel cultural, social y tecnológico, y que por supuesto inciden en los procesos de enseñanza y aprendizaje en los Smart Campus (Díaz, 2016). Esto exige el desarrollo de habilidades y destrezas en la adquisición de conocimiento y su difusión a través de canales académicos ya que, producto de la capacitación, no sólo se aprende lo asimilado directamente por la información, sino también lo que se descubre empíricamente a partir de su utilización, según las capacidades de cada uno de los miembros de la comunidad universitaria (ParraPenagos \& Rodríguez-Fonseca, 2015). Esto es independiente del pilar en el que se analice, ya que cada experiencia individual genera un aprendizaje y se convierte en una nueva oportunidad de intercambio de conocimiento tácito con fines de convertirse en explícito y valor intangible para la institución (Niebles-Núñez, Hernández-Palma \& Cardona-Arbeláez, 2016).

Respecto a los pilares identificados, cada uno abarca un área fundamental para el desarrollo y consolidación de un Campus Inteligente y se requiere del desarrollo de todos sus componentes para garantizar una correcta gestión de la innovación y, por consiguiente, del conocimiento.

Partiendo de la educación, que junto con la tecnología han logrado complementarse y llegar a espacios donde la educación tradicional presentaba falencias y numerosas barreras para su desarrollo (García, Fernández \& Duarte, 2017); se busca la adaptación de diferentes herramientas de forma armónica a los procesos de enseñanzaaprendizaje, donde se aprovechen al máximo los apoyos tecnológicos para el fortalecimiento de las estrategias docentes utilizadas hasta hace algunos años, propendiendo por la transformación pedagógica (Cujia, Jiménez \& Martelo, 2017; Coll, Onrubia \& Mauri, 2007). En cuanto al medio ambiente, la reducción en las emisiones de carbono y la búsqueda de la sostenibilidad a partir de soluciones alternativas, como el uso de las denominadas energías limpias provenientes de la radiación solar, corrientes de aire, entre otras (Casadiego-Ruiz, Pardo-García \& Díaz-Rodríguez, 2017; Vergara-Barrios, Rey-López, Osma-Pinto \& Ordóñez-Plata, 2014), ya no son tema que compete solo a las ciudades sino que abarca a todas aquellas instituciones que puedan generar un impacto significativo en la sociedad, tal y como lo son las instituciones educativas, las cuales son modelo de desarrollo para el siguiente pilar correspondiente a la sociedad, ya que desde los campus inteligentes pueden generarse soluciones replicables a ciudades, en busca a su vez, de la generación de Smart Cities (Maza, Orozco \& Ospino, 2017).

Adicionalmente, la gobernanza juega un papel estratégico en el desarrollo de los campus, por su lucha para la eliminación de barreras de implementación tecnológica, las cuales provienen principalmente de la cultura y la aversión al cambio por parte de la comunidad universitaria (Prieto, 2014). La gobernanza permite conformar un ecosistema digital que favorezca a los siguientes tres pilares, iniciando con la movilidad y su importancia en el favorecimiento de la circulación de los actores institucionales, tanto a nivel interno como externo, soportado en el uso de herramientas de análisis especializadas de origen tecnológico (Montoya, Espinal \& Vahos, 2016; Casadiego-Ruiz, 2017). El otro pilar beneficiado es la salud, como necesidad básica que necesita ser satisfecha de la mejor manera posible para garantizar una buena calidad de vida de los miembros de la comunidad, partiendo de estrategias de prevención y control de factores que puedan afectarla; y llegando a la Investigación, Desarrollo e innovación $(I+D+i)$, iniciando con procesos de Vigilancia Tecnológica 
continua e Inteligencia Competitiva que soporten de forma adecuada el proceso para la toma de decisiones (identificación, monitoreo, hallazgos, obtención, análisis, interpretación y difusión de la información) que se traduzcan en soluciones a las necesidades de la comunidad (Moya-Espinosa \& Moscoso-Durán, 2017). Esto último es válido incluso para aquellos miembros potenciales como los futuros estudiantes, de modo que adquieran las destrezas necesarias para que se desenvuelvan en los ambientes digitales que se presentan en la actualidad (Marulanda, Giraldo \& López, 2014).

Finalmente, se realizó un compendio de resultados para revisión de expertos, quienes validaron los hallazgos presentados; dicha validación fue realizada por expertos pertenecientes a la Universidad de la Habana, de Cuba, y a la Universidad Industrial de Santander, de Colombia.

\section{Conclusiones}

Los autores se permiten proponer las siguientes conclusiones como fundamento para la discusión académica:

El concepto de Smart-Campus tiene diferentes definiciones en la literatura, concordando la mayoría en que se asocia a un tipo de universidad capaz de recabar información, generalmente apoyada por tecnología, para facilitar la toma de decisiones en diferentes áreas de la gestión organizacional y del conocimiento. Se resalta que, si bien la tecnología es un eje transversalizador del proceso, no es el único y que se deben revisar otras dimensiones y categorías al momento de considerar el desarrollo y aplicación de una universidad inteligente.

Si bien cada institución cuenta con particularidades y autonomía, ya sea por sus características geográficas, socio-económicas, políticas, entre otras; es posible entender que exista un conjunto de elementos (factores) que se deben comprender y desarrollar para orientar la conformación de un campus dinámico y reflexivo, orientado al mejoramiento continuo. En años recientes, se han consolidado paradigmas tecnológicos tanto para el registro de la información, su análisis en grandes volúmenes de datos como para la generación de conclusiones de utilidad para toda la comunidad universitaria. La idea de Smart-campus se debe construir, como se observa en la literatura y en la experiencia propia de la UDES, con un proceso participativo y que integre a los diferentes actores de la organización.

La innovación y el aprendizaje son aspectos que se encuentran fuertemente vinculados. La sociedad se encuentra en una constante evolución, vista desde las nociones de conocimiento y aprendizaje; donde el trabajo colaborativo y en red de los diferentes actores institucionales (y con vínculos externos a la organización) permiten consolidar procesos innovadores.

Si bien la revisión particular de las circunstancias institucionales y su vínculo con la literatura, lleva a los autores del presente artículo a discutir un conjunto de siete pilares (educación, ambiente, sociedad, gobernanza, infraestructura y movilidad, salud y componente I+D+i); esto no significa que los mismos deban ser estáticos y estándares para otras organizaciones; los mismos deben adaptarse, reforzarse u omitirse según las fortalezas, capacidades e intereses de las diferentes instituciones.

La incorporación de elementos de inteligencia, Smart, en la Universidad, debe realizarse con parámetros de respeto a la autonomía, la intimidad y en general, a los principios que determina el ser humano en sociedad. No se debe considerar que, al motivar la integralidad en los procesos educativos, se puedan desconocer o transgredir estos elementos.

\section{Referencias}

Aion, N., Helmandollar, L., Wang, M., \& Ng, J. W. P. (2012). Intelligent campus (iCampus) impact study. Web Intelligence and Intelligent Agent Technology (WI-IAT), IEEE/WIC/ACM International Conferences 
on, WI-IAT, 291-295. doi: https://doi.org/10.1109/ WI-IAT.2012.261

Atif, Y., \& Mathew, S. (2013). A social web of things approach to a smart campus model. Proceedings IEEE International Conference on Green Computing and Communications and IEEE Internet of Things and IEEE Cyber, Physical and Social Computing, GreenCom-iThings-CPSCom, 349-354. doi: https://doi. org/10.1109/GreenCom-iThings-CPSCom.2013.77

Berghofer, R. (2013). Smart university vision presented at robert gordon university. Recuperado de: https://smartuniversity.uwl.ac.uk/blog/?p=100

Bures, V., Tucnik, P., Mikulecky, P., Mls, K., \& Blecha, P. (2016). Application of Ambient Intelligence in Educational Institutions: Visions and Architectures. International Journal of Ambient Computing and Intelligence (IJACI), 7, 27. doi: 10.4018/IJA$\mathrm{Cl} .2016010105$

Bustamante-Zapata, L. F., Porto-Pérez, I. A., \& Hernández-Taboada, F. (2013). Gestión estratégica de las áreas funcionales de la empresa: una perspectiva competitiva internacional. Revista de Investigación, Desarrollo e Innovación, 4 (1), 56-68. doi: https://doi.org/10.19053/20278306.2607

Casadiego-Ruiz, N. M. (2017). Construcción de un entorno conceptual alrededor del tema del tránsito urbano. Revista de Investigación, Desarrollo e Innovación, 7 (2), 355-365. doi: https://doi. org/10.19053/20278306.v7.n2.2017.6074

Catapult-Future Cities. (2016). Smart campus - university of glasgow. Recuperado de: http://futurecities.catapult.org.uk/project/smart-campus-university-of-glasgow/

Centre of Regional Science. (2007). Smart cities. Ranking of European medium-size cities. Viena, Austria.
Coccoli, M., Guercio, A., Maresca, P., \& Stanganelli, L. (2014). Smarter universities: A vision for the fast changing digital era. Journal of Visual Languages and Computing, 25 (6), 1003-1011. doi: https://doi. org/10.1016/j.jvlc.2014.09.007

Coll, C., Onrubia, J., \& Mauri, T. (2007). Tecnología y prácticas pedagógicas: las TIC como instrumentos de mediación de la actividad conjunta de profesores y estudiantes. Anuario de psicología, 38 (3). Recuperado de: http://www.redalyc.org/articulo. oa?id=97017407003>

Cujia, S., Jiménez, M. L., \& Martelo, R. J. (2017). Tecnologías de información y comunicación en el marco de la estrategia instruccional del docente universitario. Revista Espacios, 38 (55). doi: a17v38n55/17385501

Díaz-Bernal, J. G. (2016). Tecnología:i un desafío para salir del riesgo?. Praxis \& Saber, 7 (14), 71-90. doi: http://dx.doi.org/10.19053/22160159.5218

Donate-Manzanares, M., \& Guadamillas-Gómez, F. (2010). Estrategia de gestión del conocimiento y actitud innovadora en empresas de castilla-la mancha. un estudio exploratorio. Investigaciones Europeas de Dirección Y Economía de La Empresa, 16 (1), 31-54. https://doi.org/10.1016/S11352523(12)60002-1

Figueroa-Cuello, A. N., Pardo-García, A., \& Díaz-Rodríguez, J. L. (2017). Sistema control supervisor de clientes con acceso remoto para sistemas solares fotovoltaicos autónomos. Revista de Investigación, Desarrollo e Innovación, 7 (2), 367-378. doi: https:// doi.org/10.19053/20278306.v7.n2.2017.6104

García, R. A., Fernández, F. H., \& Duarte, J. E. (2017). Modelo de integración de las TIC en instituciones educativas con características rurales. Revista Espacios, 38 (50) 26- 40. doi: a17v38n50/17385026

García-Rubiano, M., \& Forero-Aponte, C. (2015). Contrato psicológico y cambio organizacional en 
una entidad perteneciente al sector terciario de la ciudad de Bogotá, Colombia. Revista de Investigación, Desarrollo e Innovación, 6 (1), 15-28. doi: https://doi.org/10.19053/20278306.4047

Gonzalez-Ariza, A. L., Castro, J. P., \& Roncallo, M. (2004). Diagnóstico de la gestión de conocimiento en una empresa grande de Barranquilla (Colombia). Una actividad de vinculación cooperativa universidad - sector productivo. Ingeniería y Desarrollo, (16), 70-103. doi: http://www.redalyc.org/ html/852/85216006/

Grant, R. M. (1996). Toward a knowledge-based theory of the firm. Strategic Management Journal, 17 (S2), 109-122. doi: https://doi.org/10.1002/ smj.4250171110

Hartmann, A. (2006). The role of organizational cultureinmotivating innovative behaviourinconstruction firms. Construction Innovation, 6 (3), 159-172. doi: https://doi.org/10.1108/14714170610710712

Hipwell, S. (2014). Developing smart campuses \#x2014; A working model. Intelligent Green Building and Smart Grid (IGBSG), International Conference on (IGBSG), 1-6. doi: https://doi.org/10.1109/ IGBSG.2014.6835169

Hirsch, B., \& Ng, J. W. P. (2011). Education beyond the cloud: Anytime-anywhere learning in a smart campus environment. In International Conference for Internet Technology and Secured Transactions, ICITST, 718-723. Recuperado de: https://www.scopus.com/inward/record.uri?ei$\mathrm{d}=2-\mathrm{s} 2.0-84857493677$ \&partnerlD $=40 \& \mathrm{md} 5=-$ 70255f5e549f1fb76b5ec7ea729821c3

Jazmín, C., Barrera, G., Romero, E., \& Becerra Ardila, L. E. (2015). Innovación Social en pequeñas ciudades.

Khabou, N., Rodriguez, I. B., Gharbi, G., \& Jmaiel, M. (2014). A threshold based context change detection in pervasive environments: Application to a smart campus. Procedia Computer Science, 32, 461-468. doi: https://doi.org/10.1016/j. procs.2014.05.448

Lazaroiu, G. C., Dumbrava, V., Costoiu, M., Teliceanu, M., \& Roscia, M. (2015). Smart campus-an energy integrated approach. Renewable Energy Research and Applications (ICRERA), International Conference on, 5, 1497-1501. doi: https://doi.org/10.1109/ ICRERA.2015.7418657

Liberona, D., \& Ruiz, M. (2013). Análisis de la implementación de programas de gestión del conocimiento en las empresas chilenas. Estudios Gerenciales, 29 (1), 151-160. doi: https://doi.org/10.1016/j.estger.2013.05.003

Makdissi, A., Boutaud, B., \& Dalmolin, R. (2016). Smart Systems, 59-64.

Martínez-Caro, E. (2009). La gestión del conocimiento a través del e-learning. un enfoque basado en escenarios. Investigaciones Europeas de Dirección y Economía de La Empresa, 15 (1), 29-44. doi: https://doi.org/10.1016/S1135-2523(12)60076-8

Marulanda, C. E., Giraldo, J., \& López, M. (2014). Acceso y uso de las Tecnologías de la información y las Comunicaciones (TICs) en el aprendizaje: El Caso de los Jóvenes Preuniversitarios en Caldas, Colombia. Formación universitaria, 7 (4), 47-56. doi: http:// dx.doi.org/10.4067/S0718-50062014000400006

Mattoni, B., Pagliaro, F., Corona, G., Ponzo, V., Bisegna, F., Gugliermetti, F., \& Quintero-Nunez, M. (2016). A matrix approach to identify and choose efficient strategies to develop the Smart Campus. Environment and Electrical Engineering (EEEIC), IEEE 16th International Conference on. doi: https://doi. org/10.1109/EEEIC.2016.7555571

Maza-Figueroa, N. P., Orozco-Acevedo, M. A., \& Ospino-Pinedo, M. E. D. (2017). Modelo de gestión estratégica para el desarrollo de un campus inteligente basado en conceptos de Smart City en la Uni- 
versidad de Cartagena-Campus Piedra de Bolívar. (Trabajo de pregrado Universidad de Cartagena). doi: http://190.242.62.234:8080/jspui/handle/11227/5931

Ministerio de Educación Nacional. (2017). Gestión del conocimiento en las IES: ¿Estamos preparados para ello?. Bogotá, Colombia. Recuperado de: http://www.mineducacion.gov.co/1759/articles-

Montoya, R. A. G., Espinal, A. A. C., \& Vahos, J. D. H. (2016). Transporte verde: eficiencia y reducción de CO2 integrando gestión, tecnologías de información y comunicaciones (TIC) y un metaheurístico. Producción+ Limpia, 10 (2), 53-60. Recuperado de: http://repository.lasallista.edu.co:8080/ojs/index. $\mathrm{php/pl/article/view/897/620}$

Moya-Espinosa, P. I., \& Moscoso-Durán, F. F. (2017). Vigilancia tecnológica e inteligencia competitiva en el modelo empresarial del sector hotelero colombiano. Revista De Investigación, Desarrollo E Innovación, 8 (1), 11-22. doi: https://doi. org/10.19053/20278306.v8.n1.2017.7367

Muñoz-Durán, J., Marín-Martínez, M., \&Vallejo-Triano, J. (2006). La vigilancia tecnológica en la gestión de proyectos de $\mathrm{I}+\mathrm{D}+\mathrm{i}$ : recursos y herramientas. El Profesional de La Información, 5 (5), 411-419. Recuperado de: http://eprints.rclis.org/9400/1/ vol15_6.1.pdf

Naranjo-Valencia, J. C., Jiménez-Jiménez, D., \& Sanz-Valle, R. (2012). ¿Es la cultura organizativa un determinante de la innovación en la empresa?. Cuadernos de Economía y Dirección de la Empresa, 15 (2), 63-72. doi: https://doi.org/10.1016/j. cede.2011.07.004

Niebles-Núñez, W. A., Hernández-Palma, H. G., \& Cardona-Arbeláez, D. (2016). Gestión tecnológica del conocimiento: herramienta moderna para la gerencia de instituciones educativas. Revista de Investigación, Desarrollo e Innovación, 7 (1),
25-36. doi: https://doi.org/10.19053/20278306. v7.n1.2016.5633

Nieto-Antolín, M., \& Navas-López, J. (2003). Estrategias de Innovación y Creación de Conocimiento Tecnológico en las Empresas Industriales Españolas. Madrid, España: Editorial Civitas, Ed.

Nonaka, I., \& Takeuchi, H. (1995). The Knowledge Creating Company: How Japanese Companies Create the Dynamics of Innovation. New York, NY, USA: Oxford University Press.

Pagliaro, F., Mattoni, B., Gugliermenti, F., Bisegna, F., Azzaro, B., Tomei, F., \& Catucci, S. (2016). A roadmap toward the development of Sapienza Smart Campus. EEEIC 2016 - International Conference on Environment and Electrical Engineering. doi: https:// doi.org/10.1109/EEEIC.2016.7555573

Parra-Penagos, C., \& Rodríguez-Fonseca, F. (2015). La capacitación y su efecto en la calidad dentro de las organizaciones. Revista de Investigación, Desarrollo e Innovación, 6 (2), 131-143. doi: https://doi. org/10.19053/20278306.4602

Pertuz-Peralta, V., \& Perez-Orozco, A. B. (2015). Modelo de cultura organizacional innovadora en caficultores del departamento del Cesar, Colombia. Revista de Investigación, Desarrollo e Innovación, 6 (2), 117-130. doi: https://doi. org/10.19053/20278306.3687

Pitre, I. J., Gómez, R. J. M., \& Pertuz, M. P. (2017). Diagnóstico sobre accesibilidad e integración digital dentro del sector universitario colombiano. Saber, Ciencia y Libertad, 12 (1), 225-235. Recuperado de: http://ojs.sabercienciaylibertad.com/ojs/ index.php/scyl/article/view/227/pdf_15

Prettel, M. G., Cantillo, R. R., \& Ávila, D. M. (2014). Transformación pedagógica mediada por tecnologías de la información y la comunicación (TIC). Saber, Ciencia y Libertad, 9 (2), 217-228. doi: http:// dx.doi.org/10.22525/sabcliber.2014v9n2.217228 
Prieto-Mejía, S. (2014). La Incorporación de Tecnologías Educativas en la Universidad del Magdalena bajo un enfoque de Smart Campus. Recuperado de: http://dspace.redclara.net/bitstream/10786/723/1/34-La\%20Incorporaci\%C3\%B3n\%20de\%20Tecnolog\%C3\%ADas\%20Educativas\%20en\%20la\%20Universidad\%20del\%20 Magdalena $\% 20$ bajo\%20un\%20enfoque $\% 20$ de\%20Smart\%20Campus.pdf

Smith, K. G., Collins, C. J., \& Clark, K. D. (2005). Existing Knowledge, Knowledge Creation Capability, and the Rate of New Product Introduction in High-Technology Firms. The Academy of Management Journal, 48 (2), 346-357. doi: https://doi. org/10.2307/20159660

Szabo, R., Farkas, K., Ispany, M., Benczur, A. A., Batfai, N., Jeszenszky, P., \& Feher, G. (2013). Framework for smart city applications based on participatory sensing. In 4th IEEE International Conference on Cognitive Infocommunications, CogInfoCom 2013 - Proceedings, 295-300. doi: https://doi.org/10.1109/ CogInfoCom.2013.6719260

Tan, J. (2006). Growth of industry clusters and innovation: Lessons from Beijing Zhongguancun Science Park. Journal of Business Venturing, 21 (6), 827-850. doi: https://doi.org/10.1016/j.jbusvent.2005.06.006
Tan, L., \& Wang, N. (2010). Future Internet: The Internet of Things. In ICACTE 2010 - 2010 3rd International Conference on Advanced Computer Theory and Engineering, Proceedings 5, V5376-V5380. doi: https://doi.org/10.1109/ICACTE.2010.5579543

Valdelamar-Zapata, J. A., Ramírez-Cruz, Y. L., Rodríguez-Rivera, P. D., \& Morales-Rubiano, M. E. (2015). Capacidad innovadora: cómo fomentarla, según docentes de Ciencias Económicas e Ingeniería de la UMNG. Revista de Investigación, Desarrollo e Innovación, 6 (1), 7-14. doi: https://doi. org/10.19053/20278306.3454

Vargas, F., \& Castellanos, O. (2005). Vigilancia como herramienta de innovación y desarrollo tecnológico. Caso de aplicación: Sector de empaques plásticos flexibles. Ingeniería $e$ investigación, 25 (2). Recuperado de: http://www. scielo.org.co/scielo.php?script=sci_arttext\&pi$\mathrm{d}=\mathrm{S} 0120-56092005000200004 \& \mathrm{nrm}=\mathrm{iso}$

Vergara-Barrios, P. P., Rey-López, J. M., Osma-Pinto, G. A., \& Ordóñez-Plata, G. (2014). Evaluación del potencial solar y eólico del campus central de la Universidad Industrial de Santander y la ciudad de Bucaramanga, Colombia. Revista UIS Ingenierías, 13 (2), 49-57. Recuperado de: http://revistas.uis. edu.co/index.php/revistauisingenierias/article/ view/49-57 